\title{
La pauvreté en Poitou sous l'Ancien Régime vue à travers le prisme de l'hôpital général de Poitiers
}

Emmanuel Thévenet

\section{(2) OpenEdition}

\section{Journals}

Édition électronique

URL : http://journals.openedition.org/abpo/550

DOI : 10.4000/abpo.550

ISBN : 978-2-7535-1504-8

ISSN : 2108-6443

Éditeur

Presses universitaires de Rennes

Édition imprimée

Date de publication : 30 décembre 2006

Pagination : 159-182

ISBN : 978-2-7535-0405-9

ISSN : 0399-0826

\section{Référence électronique}

Emmanuel Thévenet, «La pauvreté en Poitou sous l'Ancien Régime vue à travers le prisme de l'hôpital général de Poitiers ", Annales de Bretagne et des Pays de l'Ouest [En ligne], 113-4 | 2006, mis en ligne le 30 décembre 2008, consulté le 30 avril 2019. URL : http://journals.openedition.org/abpo/550 ; DOI :

$10.4000 / a b p o .550$ 


\title{
La pauvreté en Poitou sous l'Ancien Régime vue à travers le prisme de l'hôpital général de Poitiers
}

\author{
Emmanuel THÉVENET \\ Professeur d'histoire-géographie, lycée Marcelin-Berthelot (Châtellerault) \\ Université de Poitiers
}

Pour saisir la pauvreté poitevine sous l'Ancien Régime, l'analyse des populations accueillies dans l'hôpital général de Poitiers constitue une approche historique relativement pertinente. En effet, par les documents qu'il a produits, l'hôpital nous renseigne sur les caractéristiques sociales, économiques et démographiques des pauvres poitevins. Néanmoins, cette source d'informations et de réflexion doit être relativisée à plus d'un égard. La pauvreté n'est saisie que par le hasard des sources conservées. Cellesci sont parfois lacunaires et, souvent, ne renseignent pas l'historien sur les questions qu'il se pose. Par ailleurs, il est hasardeux de réfléchir sur un phénomène aussi composite que la pauvreté à partir d'une source unique. La complexité de ce fait social mène constamment l'historien à jointoyer les éléments de la mosaïque avec d'autres mortiers que l'approche quantitative. Ainsi, le recours aux écrits de "théoriciens " tels que Michel Foucault ${ }^{1}$, ou d'érudits locaux (tels que Pierre Rambaud ${ }^{2}$ ) permet une approche stimulante. La lecture des écrits contemporains, et notamment ceux des philosophes des Lumières, permet d'intégrer les représentations mentales des hommes cultivés et intellectuellement dominants des XVII et XVIII ${ }^{\mathrm{e}}$ siècles.

Le présent article est extrait d'un mémoire de maîtrise consacré à l'hôpital général de Poitiers ${ }^{3}$, qui avait pour but d'analyser non pas les comportements de la population pauvre en Poitou mais plutôt la création, le

1. FoucAutr, Michel, Histoire de la folie à l'âge classique, Paris, Gallimard, coll. « Tel ", 1976.

2. Rambaud, Pierre, L'Assistance publique à Poitiers jusqu'à l'an V, Poitiers, MSAO, $3^{\text {e }}$ série, tome $5,1911$.

3. THÉvenET, Emmanuel, L'Hôpital général de Poitiers à l'âge classique, 1659-1789, mémoire de maîtrise sous la direction de Fabrice Vigier, Poitiers, 2004. 
fonctionnement et la rupture anthropologique que constitue un hôpital général dans une ville de province.

\section{Rapide présentation de l'institution}

La fondation de l'hôpital général de Poitiers se situe en 1657. Celle-ci répond à la volonté de la ville de voir se mettre en place une structure susceptible d'accueillir et d'encadrer les populations marginales qui se pressent dans les rues de la cité. Néanmoins, une telle entreprise prend du temps, l'ensemble des structures bureaucratiques de la monarchie ayant un rôle à jouer dans le processus décisionnel. Entre la fondation officielle de l'hôpital, et les lettres patentes qui lui sont octroyées par le roi, dix-huit ans passent, ce qui n'empêche pourtant pas cette institution charitable de fonctionner, comme l'attestent les registres des délibérations du bureau ${ }^{4}$.

Cet établissement apparaît comme la conjugaison de deux desseins corrélés et probablement concurrents. Les notables de Poitiers ont la volonté de créer un hôpital général dans leur ville, mais doivent s'en remettre à la puissance royale pour asseoir la fondation. Ce faisant, les Poitevins abolissent une part de leur liberté de décision, en échange d'un affermissement du pouvoir de l'hôpital dans les domaines économique, judiciaire et réglementaire. Ainsi, l'hôpital s'inscrit-il dans un processus d'ampleur nationale, visant à réprimer la pauvreté et la mendicité dans tout le royaume de France. C'est ce grand cadre institutionnel qui donne à l'hôpital poitevin une partie de ses moyens de subsistance, notamment par l'union forcée de maladreries et d'aumôneries. L'établissement possède une certaine autonomie financière, qui se traduit par l'existence de budgets exécutés par le bureau et par lui seul. En outre, la mise en place de la manufacture procède à la fois de cette volonté d'indépendance et de la recherche d'une pédagogie de l'enfermement : les pauvres doivent être rééduqués par le travail et la piété.

Cette pédagogie passe aussi par une symbolique de l'enfermement adressée aux pauvres comme aux habitants de la ville. Établi dans des locaux qui lui sont propres, à la limite de la ville, l'hôpital est une zone frontière au sens social et au sens spatial du terme, dans laquelle une vie de semi-autarcie s'ébauche. Pour autant, tous les liens avec le monde extérieur ne sont pas coupés. Le rôle que s'assigne l'hôpital empêche une mise

4. Les papiers relatifs à l'hôpital général de Poitiers ont été déposés aux Archives départementales de la Vienne et aux Archives municipales de Poitiers (médiathèque François-Mitterrand). Aux Archives départementales de la Vienne, ces documents sont, pour l'essentiel, contenus dans le dépôt 115, dans les portefeuilles II. A, II. B, II. D1 et II. E2 à II. E7. En outre, trois registres de délibérations et les comptes de recettes et de dépenses ont été classés à la cote J 213. Par ailleurs, un plan de l'hôpital général, dressé en 1800, est conservé sous la cote D 26. Aux Archives municipales de Poitiers sont conservés les registres de sépultures de 1704 à la fin de la Révolution, sous les cotes 270, 271 et 273. Enfin, il convient de préciser que les Archives hospitalières semblent conserver des documents et des objets relatifs à l'hôpital général. Mais nos démarches pour tenter d'y avoir accès sont restées vaines. 
à l'écart totale. Les pauvres, en son sein, se trouvent placés dans une sorte de sas, dans lequel ils doivent apprendre à mener une vie perçue comme honnête, intégrée et " normale ». Dès lors, il est utile de s'interroger sur la perception de la pauvreté par les élites intellectuelles de l'époque.

\section{La pauvreté perçue : le regard des élites}

Il y a une rupture éminente et essentielle au XVII ${ }^{\mathrm{e}}$ siècle. Une des manifestations de ce changement de civilisation est le " grand renfermement". Elle continue de se diffuser tout au long du XVIII ${ }^{\mathrm{e}}$ siècle et entraîne avec elle les Lumières, la Révolution, l'Empire. Ce que les historiens nomment " époque moderne " connaît une double naissance : le XVI ${ }^{\mathrm{e}}$ siècle, avec la Renaissance, l'Humanisme, la Réforme et les guerres de religion, qui posent les bases de l'État dit "moderne", et le XVII siècle qui lui donne les moyens de s'incarner dans une idée faite corps, le " pré carré " français. Un glissement sémiologique s'opère. Il est tout particulièrement perceptible dans le domaine de la pauvreté. Sa conception sociale se transforme, en même temps que se modifie toute la vision de l'homme et du monde. En rendant l'homme seul responsable de ses choix, les humanistes créent une nouvelle perception des actions individuelles. Néanmoins, cette solitude ne sous-entend pas une liberté totale d'action. Chacun est libre à l'intérieur de cercles concentriques qui limitent la liberté de mouvement, mais ne l'annihilent pas totalement. Quant au pauvre, il n'est plus l'élu de la puissance divine. Il devient un être marginal, qui ne veut pas s'intégrer à la nouvelle société qui se fait jour. Dès lors, derrière un vocabulaire hérité du Moyen Âge (" pauvres ", " mendiants ", " gueux ", " gueuserie ", " gens sans aveu " "), qui en apparence ne change pas, se met en place une perception sociale en pleine mutation. Par conséquent, l'hôpital général ne peut être perçu comme une institution charitable comparable aux aumôneries du passé. Sa signification sociale est plus profonde et plus complexe.

La pauvreté, sous l'Ancien Régime comme de nos jours, est un phénomène difficile à appréhender. La définition la plus simple est une définition négative. Être pauvre, c'est ne pas être riche. Au demeurant, une telle réponse n'est pas satisfaisante, car il existe des individus, qui, sans être des riches, n'entrent pas dans la catégorie des pauvres. Mais derrière cette explication frustrante et inadéquate, se cache le concept central de la notion d'indigence et de misère : le pouvoir économique des agents sociaux. Pour autant, cette situation centrale ne signifie pas unique. Pour reprendre la définition de Guy Cabourdin et Georges Viard, être pauvre sous l'Ancien Régime

5. Le mot " pauvre ", apparaît en 1050 dans Alexis, et est écrit "povre ". La forme actuelle est née au ${ }^{\mathrm{er}}$ siècle, avec au repris au latin "pauper». C'est aussi dans Alexis que se forme " gens ", issu du latin "gens ", "gentis ". Le terme " mendiant " se présente en 1170 dans Floire et Blancheflor, éliminant l'ancien français "mendi " du latin "mendicus ". Quant au substantif "gueux ", il est dû à Esnault, au XIV siècle, avant d'être repris par François Villon en 1452 (DAuZAT, Albert, Dubors, Jean et MitTERAND, Henri, Dictionnaire étymologique et historique du français, Paris, Larousse, 1994). 
c'est être " celui qui souffre, qui est humble, affligé, dans le malheur. Dans une optique plus restreinte, il est celui qui se trouve dans la "nécessité", qui n'a que son travail pour vivre, qui faute de réserves, a la hantise du pain quotidien. Le pauvre n'est pas totalement sans ressource; il peut avoir son instrument de travail, même un petit bien-fonds. Le plus souvent, le mot " mendiant " sert à désigner celui qui est tombé dans la pauvreté. En fait, de la pauvreté à la mendicité, la différence n'est pas de nature, mais de degré ${ }^{6}$ ". De nouvelles notions apparaissent : souffrance sociale, physique, morale et humilité de la condition de l'individu dans le monde. Pour essayer de cerner davantage ce concept de pauvreté, nous nous appuierons sur les définitions données par les hommes du temps, à travers les dictionnaires, les encyclopédies, les ouvrages théoriques ou les traités.

Le problème de la pauvreté implique une réponse sociale. C'est l'action menée par les hôpitaux généraux qui la constitue. Néanmoins, la solution n'est pas accueillie avec bienveillance par tous : de fortes critiques se sont élevées, notamment chez les philosophes des Lumières, contre ces institutions. Enfin, il s'agit d'organiser un dépassement du couple pauvreté/hôpital général. Il faut trouver une articulation susceptible d'expliquer pourquoi la société de l'époque moderne met en place une telle structure d'enfermement. Cette attitude morale et politique vis-à-vis de la pauvreté et de la marginalité est révélatrice d'un état d'esprit nouveau qui se développe à l'âge classique et au siècle des Lumières.

Le jugement porté par les élites du XVIII siècle est teinté d'une forte coloration morale. Le " vrai " pauvre, comme le " vrai mendiant ", est toléré mais il ne peut s'agir que d'un enfant abandonné, d'un adulte réduit à l'indigence par un accident de la vie ou d'un vieillard, incapable de subvenir à ses besoins. Par contre, le mendiant de " profession " est perçu très négativement, car il se laisse porter par l'effort public. Installé dans l'oisiveté, il ne vit que par la charité du public et les structures mises en place par la monarchie. Il y a donc bien un glissement sémantique qui s'opère à l'âge classique. La pauvreté est subdivisée en catégories spécifiques, qui regroupent des individus ayant en commun le fait de vivre petitement, mais pour des raisons qui diffèrent. La moralité de l'âge de la Raison ne peut tolérer la marginalité d'individus qui survivent sans travailler, tandis qu'aucune excuse ne peut justifier leur " oisiveté ", assimilée à de la paresse, voire à de la malveillance sociale. Comme le remarque Sébastien Jahan, « il y aurait une mendicité "légitime", celle des "indigents invalides ou infortunés qui, ne pouvant se procurer le nécessaire par leur travail, n'ont d'autres ressources pour subsister que la charité publique", et, à l'opposé, une mendicité "illégitime", celle des "vagabonds et fainéants de professions "7" ". Mais

6. CABOURDin, Guy et VIARD, Georges, Lexique historique de la France d'Ancien Régime, Paris, Colin, 1978, p. 256, article «Pauvreté ».

7. JAHAN, Sébastien, "Approches qualitatives de la pauvreté en haut Poitou au xVIII ${ }^{\mathrm{e}}$ siècle ", $B S A O, 5^{\mathrm{e}}$ série, tome $\mathrm{V}, 2^{\mathrm{e}}$ trimestre 1991, p. 104. L'auteur renvoie aux travaux du comité de mendicité. 
cette malveillance individuelle n'empêche pas, dans l'esprit des philosophes notamment, une certaine malveillance d'État, organisée dans les fondations hospitalières.

L'enfermement est une création institutionnelle du XVII ${ }^{\mathrm{e}}$ siècle. Celle-ci est un marqueur de la rupture décisive qui s'opère lors du Grand Siècle, rupture déjà mise en évidence par Michel Foucault et Michel de Certeau. L'enfermement apparaît à la fois comme une invention économique et sociale, mais aussi comme le symptôme de l'évolution, à l'époque moderne, de la morale chrétienne. La pauvreté, au Moyen Âge, n'est pas encouragée, mais tolérée parce qu'elle met en acte la vie du Christ. À l'époque moderne, après la Réforme, la pauvreté volontaire apparaît comme une révolte contre la société entière. Si la Réforme est le point de départ d'une première laïcisation de l'Europe, le traitement réservé à la pauvreté montre le glissement opéré par la pensée religieuse, notamment catholique, pour la fixer à la limite du profane et du sacré. La rébellion du pauvre contre le travail est aussi une rébellion contre Dieu. Les perceptions de la pauvreté et de la charité du siècle des Lumières sont le fruit de cette longue décantation intellectuelle, réalisée sur près de trois siècles, par des penseurs aux formations culturelles finalement voisines et connexes, car enchevêtrées les unes avec les autres : la pensée réformée entraîne la Contre Réforme, qui nourrit les critiques des Lumières. Cette longue réflexion nous donne une indication sur la permanence idéologique de l'époque moderne, sur son homogénéité morale et sur l'importance du temps long, y compris dans l'histoire des idées.

Une telle analyse se place en rupture avec une étude fondée essentiellement sur des sources concrètes et tangibles. Pourtant, son économie constituerait une mystification. L'enfermement ne peut se réduire à des données matérielles, qui déterminent des budgets ou des séries statistiques : le cheminement intellectuel de plusieurs générations de penseurs serait laissé de côté. Dès lors, il nous faut considérer, avec Michel de Certeau, que « [...] chacune de ses moitiés dit de l'autre ce qui lui manque plutôt que sa vérité ${ }^{8} "$.

\section{Tentative d'appréhension de la pauvreté vécue}

Pour réaliser cette étude, nous disposons de deux sources essentielles : les registres de délibérations du bureau de l'hôpital et les registres de sépultures de l'hôpital ${ }^{9}$. Ces documents contiennent des informations relatives aux pauvres admis à l'hôpital : dans le meilleur des cas, leur nom, leur sexe,

8. CERTEAu, Michel de, La Possession de Loudun, Paris, Gallimard-Julliard, 1970, coll. " Archives ", p. 7.

9. Arch. dép. de la Vienne, dépôt 115, portefeuilles II. E2 à E5, registres des délibérations 1679-1710, 1777-1785, 1786-13 prairial an V; ADV, J 213, registres des délibérations 1700-1703, 1710-1715, 1715-1722 et 1748-1755. Arch. mun. de Poitiers (médiathèque François Mitterrand), registres de sépultures 270, 271 et 273. 
leur âge, leur origine géographique et des informations diverses telles que leur métier, leur état matrimonial ou leur qualité sont connus. En outre, il est nécessaire de préciser que les registres des délibérations de l'hôpital ne contiennent pas exclusivement des informations relatives aux pauvres, ce qui oblige les administrateurs à classer les pauvres selon qu'ils entrent, sortent ou meurent à l'hôpital.

Toutes ces données nous permettent de réaliser une étude globale sur la pauvreté accueillie à l'hôpital général de Poitiers. Néanmoins, la collection des registres est incomplète : il faut donc travailler sur des périodes isolées, comme autant de sondages dus aux hasards de la conservation des documents. En outre, les informations sur le nombre des pauvres au sein de l'hôpital sont essentiellement constituées de flux d'entrées et de sorties. En effet, le relevé des pauvres internés n'existe que pour quelques années. Il s'agit donc d'établir par l'analyse démographique, géographique et sociale de la pauvreté quels sont les grands traits de ce fait social de l'Ancien Régime.

\section{Analyse démographique}

Pour réaliser une étude démographique de la pauvreté, il faut étudier les admissions et les sorties survenues à l'hôpital général, entre 1659 et 1789 , d'après les registres de délibérations évoqués plus haut ${ }^{10}$. Le graphique qui suit synthétise les données que nous avons extraites du corpus dont nous disposons aux Archives départementales. Néanmoins, il faut garder à l'esprit que ces données sont partielles, car tous les registres ne nous sont pas parvenus.

Graphique 1 - Entrées et sorties de l'hôpital général, 1659-1789

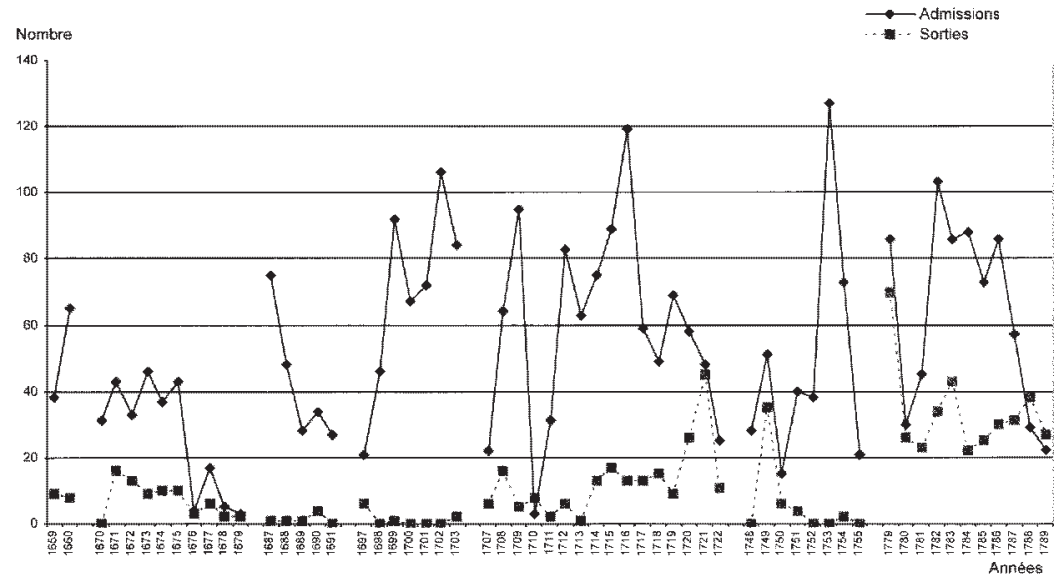

10. Les données que nous nous proposons d'étudier ne sont pas continues. 
Ce graphique laisse apparaître des phénomènes assez contradictoires. Tout d'abord, il ne semble pas y avoir de régularité dans les entrées et les sorties de l'hôpital. On observe ainsi de brusques augmentations des admissions en 1699, 1702, 1709 ou 1753. Parallèlement, le nombre d'admis chute tout aussi soudainement en 1676,1707, 1710 et 1755. En ce qui concerne les sorties, il est remarquable que leur quantité soit toujours inférieure à celle des entrées. Néanmoins, le fait de sortir, volontairement ou non, de l'hôpital n'est pas la seule façon de laisser sa place à de nouveaux admis : la mort fait aussi des ravages dans l'établissement. La mortalité fera l'objet d'une étude spécifique. Les maxima de sorties sont atteints en $1721,1749,1779$ et 1783 . Pour ces années, il est notable que le nombre des sorties soit proportionnel à celui des entrées : les administrateurs de la fin du $\mathrm{XVIII}^{\mathrm{e}}$ siècle libèrent-ils de la place lors de périodes caractérisées par une forte pression de la part des entrants?

De fait, au fil des sections de temps déterminées par les sources disponibles, il est possible de réaliser des moyennes annuelles pour chaque période.

Tableau 1 - Moyenne annuelle des entrées et sorties à l'hôpital général

\begin{tabular}{|c|c|c|c|c|c|c|c|}
\cline { 2 - 8 } \multicolumn{1}{c|}{} & \multicolumn{7}{c|}{ Moyenne annuelle } \\
\hline Périodes & $1659-1660$ & $1670-1679$ & $1687-1691$ & $1697-1703$ & $1707-1722$ & $1748-1755$ & $1779-1789$ \\
\hline Entrées & 51,5 & 26,2 & 42,4 & 69,7 & 59,5 & 49,1 & 64 \\
Sorties & 8,5 & 7,2 & 1,4 & 1,3 & 12,9 & 5,8 & 33,5 \\
\hline
\end{tabular}

Il apparaît que les moyennes d'entrées et de sorties de l'hôpital ne semblent pas obéir à une logique précisément identifiable. Si l'on constate bien une forte progression des chiffres à partir de 1687 et jusqu'en 1703, c'est-àdire lors du retournement de conjoncture de la fin du règne de Louis XIV, et à la fin du XVIII ${ }^{\mathrm{e}}$ siècle, il est pour autant difficile de corréler ces données avec des faits clairement établis. En outre, c'est pour cette dernière période que s'observe la plus importante moyenne relative aux sorties de l'hôpital. Comme le montre le graphique 2 , la hausse des prix des grains n'est pas toujours en relation avec les évolutions des flux d'admis à l'hôpital.

Ce graphique, réalisé grâce aux registres de délibérations et aux travaux de M. Duffaud ${ }^{11}$, met en relief les incohérences d'une hypothèse qui énonce que les entrées de pauvres dans les hôpitaux (et par conséquent l'extension de la pauvreté) sont un des symptômes les plus visibles des difficultés de subsistance. En effet, si cette théorie semble confirmée pour les années 1709, 1713 et 1720, voire pour 1782 (mais avec un retard de douze mois entre la hausse du prix du méteil et celle du nombre des entrées), elle est totalement infirmée en $1677,1702,1785$ et surtout 1789. Dès lors, il faut conclure que les explications conjoncturelles de la pauvreté saisie lors de

11. DufFAUD, M., Note sur le prix des grains à Poitiers depuis trois siècles, Paris, Imprimerie Impériale, 1861. 
l'entrée à l'hôpital général ne peuvent que s'insérer dans une analyse plus large et surtout plus structurelle de la société française (voire européenne) de l'époque moderne.

\section{Graphique 2 - Admissions à l'hôpital général et prix du méteil, 1659-1789}

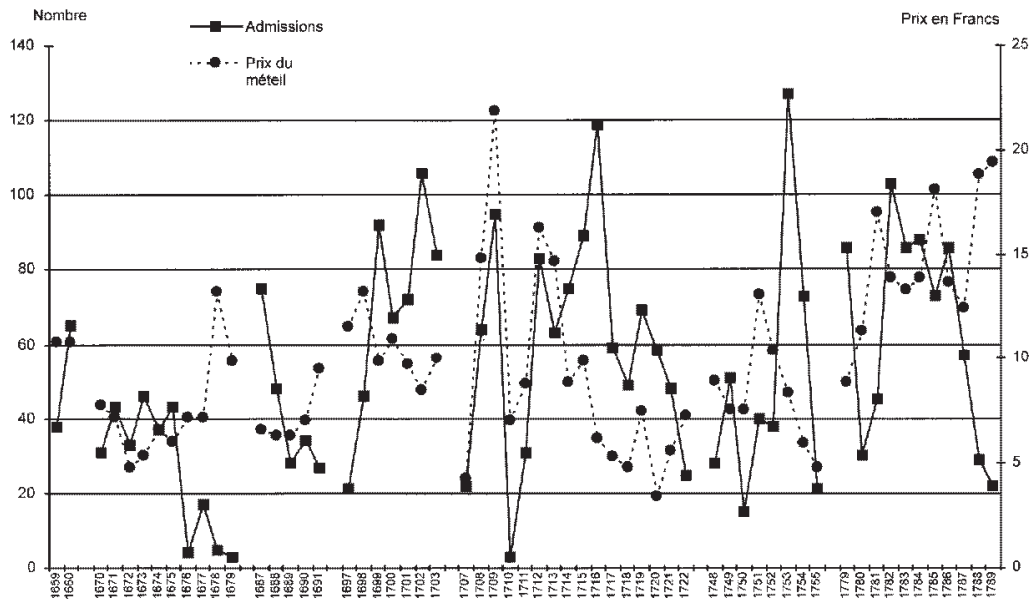

En ce qui concerne la mortalité au sein de l'hôpital, nous disposons de données fournies par les registres de sépultures de l'hôpital, pour la période $1713-1789^{12}$. Ces registres mortuaires, rédigés par les prêtres qui se sont succédé à l'intérieur de l'hôpital, constituent une source essentielle, car relativement complète. Dans le meilleur des cas, le nom, l'âge, l'origine géographique et sociale des individus sont indiqués. Pour essayer de cerner cette mortalité, et d'en définir les caractéristiques les plus prégnantes, nous analyserons d'une part les chiffres de la mort dans l'hôpital, d'autre part le nombre des victimes selon leur qualité.

Les graphiques qui suivent, construits d'après les valeurs brutes contenues dans les registres, mettent en évidence les crises de mortalités survenues à l'hôpital pendant 76 ans. Pour essayer de rendre plus lisibles ces phénomènes, nous avons utilisé la méthode d'analyse algébrique définie par Jacques Dupâquier. Les deux graphiques infra reprennent donc les mêmes données brutes, mais dans le cas du second, celles-ci ont subi un traitement statistique, susceptible de nous fournir des indications sur la gravité des crises rencontrées.

Les données brutes laissent apparaître une crise grave en 1739. Cette année semble marquer la fin de perturbations qui ont commencé en 1725,

12. Arch. mun. de Poitiers (médiathèque François Mitterrand), registres de sépultures $270,271,273$. 
Graphique 3 - La mortalité en données brutes (méthode P. Goubert)

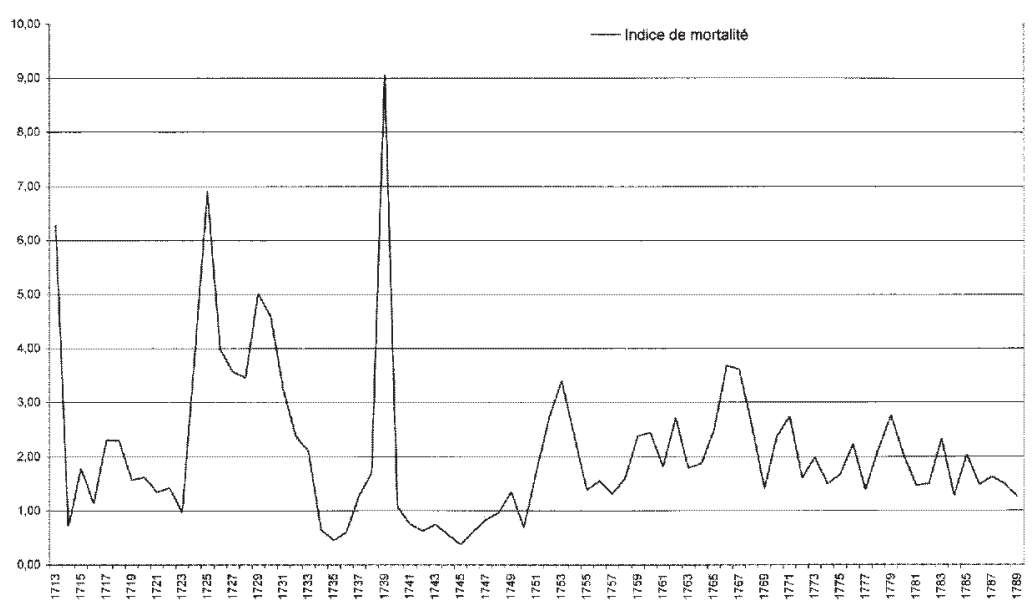

avec une brusque montée de la mortalité : soit 8,5 fois plus de morts qu'en 1723. Mais cette crise de 1725 n'est rien en comparaison du pic de mortalité atteint en 1739. On compte alors 752 morts, soit 5,1 fois plus qu'en 1725 . Cette brusque montée apparaît comme un embrasement aussi subit que limité dans le temps : en 1740, on ne compte plus que 84 morts. Néanmoins, l'année 1739 pose problème. En effet, elle constitue la dernière grande crise de type classique que connaît la France au XVIII ${ }^{\mathrm{e}}$ siècle. Pourtant, le prix du méteil, en francs, pour cette année s'établit à 8,17 francs, soit deux francs de moins qu'en 1738. Il semble donc que les effets de la hausse des prix du grain soient étalés dans le temps. En outre, les données fournies sur ces prix ne tiennent pas compte des variations saisonnières qui peuvent gravement influencer la vie quotidienne des individus les plus pauvres, souvent dans l'incapacité de constituer des stocks de nourriture en période de prix bon marché. Ce sont ces personnes pauvres qui fournissent les plus gros bataillons d'individus admis à l'hôpital. Il faut alors concevoir que sur des corps affaiblis par des privations continues, la promiscuité dans des locaux mal adaptés pour recevoir un trop grand nombre d'indigents favorise l'apparition d'épidémies. Il en est une signalée pour cette année 1739; les historiens l'identifient à une dysenterie. Malheureusement, les informations dont nous disposons dans les registres de sépultures ne signalent pas d'épidémie en 1739. Les registres des délibérations, qui auraient pu fournir des indications sur l'origine de cette crise ont disparu. L'année 1739 est donc une année noire. Elle semble pourtant clore un chapitre de l'histoire de la mortalité au sein de l'hôpital. Ce moment dramatique passé, le nombre de morts entre 1740 et 1789 s'établit dans une fourchette comprise entre 16 et 102 morts par an, c'est-à-dire 54 morts par an, en moyenne.

Néanmoins, cette analyse doit être affinée par une réflexion, non plus sur des données brutes, mais sur des indices. 
Graphique 4 - La mortalité à l'hôpital général représentée selon la méthode J. Dupâquier (calcul des indices de mortalité)

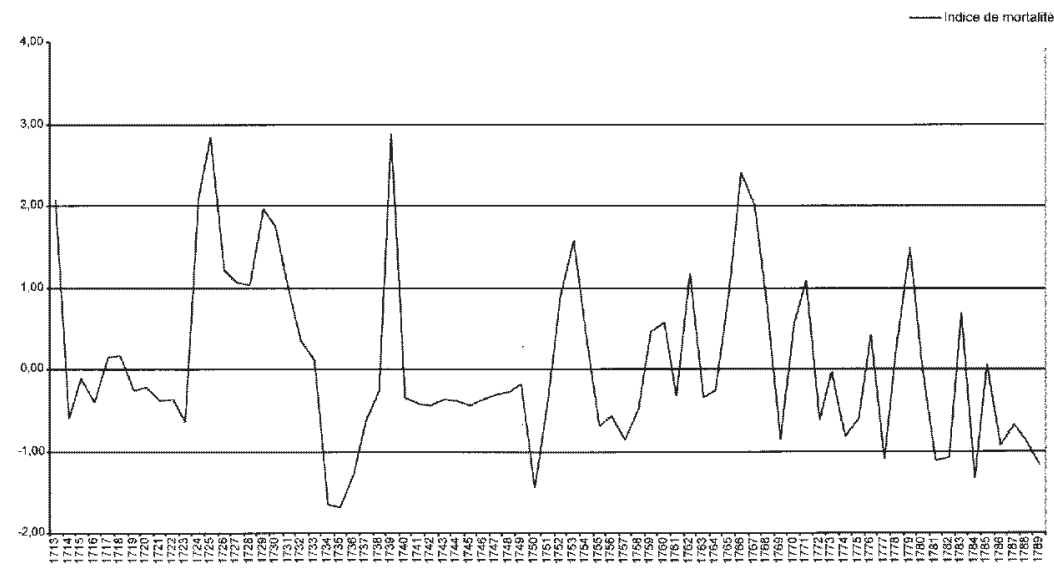

Les données contenues dans le graphique 4 appellent une explication quant au traitement que les données brutes ont connu pour constituer de telles valeurs.

La formule établie par Jacques Dupâquier s'établit comme suit ${ }^{13}$ :

$$
\mathrm{I}=(\mathrm{D}-\mathrm{M}) / \Sigma
$$

où I représente l'indice de l'année $n, \mathrm{D}$, le nombre de décès pour l'année $n$, $\mathrm{M}$, la moyenne des dix années précédentes, et $\Sigma$ l'écart type des décès pendant ces dix années.

À partir de cette équation, Jacques Dupâquier propose un indice de l'intensité des crises, selon la mortalité, établi sur le barème suivant :

Indice compris entre 1 et 2 : crise mineure

Indice compris entre 2 et $4:$ crise moyenne

Indice compris entre 4 et $8:$ crise forte

Indice compris entre 8 et $16:$ crise majeure

Indice compris entre 16 et 32 : grande crise

Indice compris supérieur à 32 : catastrophe

Avec une telle grille de lecture, la perception de la mortalité entre 1713 et 1789 change radicalement. La crise majeure (en données brutes) de 1739 se trouve singulièrement relativisée par les crises moyennes de 1713, 1725 et 1766. Par ailleurs, la crise de l'année 1739 se trouve ramenée elle aussi au rang de crise moyenne. De fait, on observe une terrible régularité des crises de mortalité tout au long des 76 années considérées. Tous les quinze ans, en moyenne, on constate une montée de la mortalité, dans le cadre de crises moyennes, ou mineures comme en 1753 et 1779. Il semble donc qu'il

13. CABOURDin, Guy et ViaRD, Georges, Lexique..., op. cit., p. 95, article : "Crise démographique ". 
y ait une fatalité de la mortalité tout au long du XVIII ${ }^{\mathrm{e}}$ siècle, celle-ci est plus modérée que lors du siècle précédent, mais très régulière par ailleurs.

Pour cerner davantage la démographie de la population admise à l'hôpital, il est nécessaire d'étudier la structuration par âge des individus, ainsi que leur sexe. Le tableau qui suit donne pour chaque période considérée l'âge moyen et l'âge médian des pauvres admis à l'hôpital.

Tableau 2 - Âges moyens et médians des pauvres admis à l'hôpital, 1670-1789

\begin{tabular}{|c|c|c|}
\hline Années & Âge moyen & Âge médian \\
\hline $1670-1673$ & 39 & 14 \\
$1674-1675$ & 46 & 57,5 \\
$1675-1679$ & 32 & 12,5 \\
$1700-1703$ & 17 & 10 \\
$1710-1715$ & 29 & 13,5 \\
$1715-1722$ & 24 & 13 \\
$1748-1755$ & 41 & 40 \\
$1777-1785$ & 46,5 & 60 \\
$1786-1789$ & 40 & 50 \\
\hline
\end{tabular}

Les données contenues dans ce tableau concernent l'âge des individus lors de leur entrée à l'hôpital. En effet, il s'agit d'étudier quels sont les individus qui doivent venir vivre au sein de l'hôpital général. Il apparaît que la structure par âges est en constante mutation. Son analyse révèle que la population admise à l'intérieur de l'hôpital est composite. Ainsi, en 16701673, si 50 \% des pauvres enfermés ont moins de 14 ans, l'âge moyen des pauvres est de 39 ans, ce qui signifie que des individus d'âge très mûr sont internés. C'est le cas, par exemple d'un certain Le Conté, admis en juin 1670 et âgé de 96 ans. Mais on constate également que, périodiquement, la population hospitalisée peut être très jeune. Ainsi, entre 1700 et 1703, 50 \% des individus ont moins de 10 ans, et l'âge moyen de ces mêmes individus est de 17 ans. Ceci est la marque d'une pauvreté qui touche surtout enfants et adolescents. C'est le cas d'un garçon admis en mars 1703 et âgé de 12 ans seulement. Néanmoins, ces données doivent être prises avec prudence. En effet, les registres de délibérations qui renferment ces informations ne sont pas toujours très au fait de l'âge des pauvres. De ce fait, nous travaillons sur des données biaisées et partielles. Pour autant, dans le cas de la période 1700-1703, les fréquentes remarques précisant l'entrée d'une " mère et ses enfants " montrent la grande jeunesse de la population renfermée à l'époque.

De fait, à la fin du XVIII ${ }^{\mathrm{e}}$ siècle, les registres deviennent plus précis quant à l'âge des pauvres. On remarque alors que la pauvreté semble se déplacer des jeunes vers les vieux, avec un maximum atteint entre 1777 et 1785, où l'âge moyen s'établit à 46 ans et demi et l'âge médian à 60 ans. La structure de la population enfermée en mutation semble signifier que la structure de la pauvreté elle-même se modifie. Il s'avère que l'hôpital se rap- 
proche de la mission que lui ont assignée les philosophes des Lumières, à savoir accueillir avant tout des vieillards, des victimes du sort et des enfants, et non pas des "fainéants " dans la force de l'âge.

Après avoir étudié la structure par âge des pauvres enfermés, il faut, pour achever la représentation démographique de la pauvreté, analyser la répartition par sexe des individus accueillis à l'hôpital. Le tableau qui suit donne le nombre d'hommes et de femmes qui ont vécu à l'hôpital général entre 1670 et 1789 .

\section{Graphique 5 - Part relative des hommes et des femmes à l'hôpital général, 1670-1789}

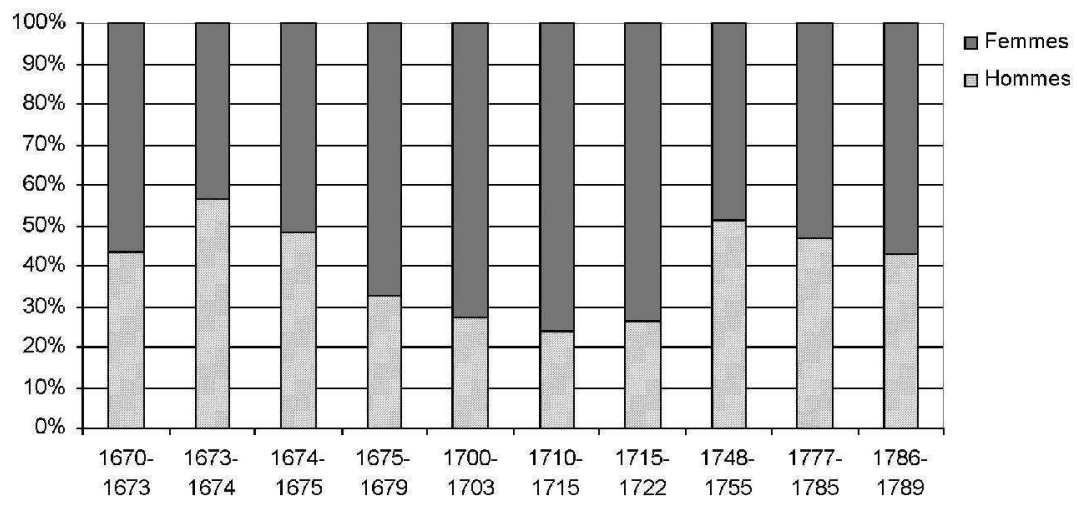

À la lecture de ce graphique, on constate l'importance des femmes dans la population hospitalisée. Minoritaires en 1673-1674 et 1748-1755 puisqu'elles ne constituent respectivement que $43,4 \%$ et $49,9 \%$ des pauvres renfermés, les femmes pauvres sont prédominantes durant toutes les autres périodes, avec un maximum atteint en 1710-1715. Il semble que cette situation soit assez facilement explicable. En effet, les femmes sont plus durement atteintes par la pauvreté que les hommes, surtout en milieu urbain. Les veuves, notamment celles ayant des enfants, comme Marie Massé admise le 10 septembre 1673 avec ses deux enfants, n'ont pas d'autre recours que la mendicité pour assurer leur survie et celle de leur famille. Celle-ci étant interdite et conduisant à l'hôpital général, les veuves y sont très présentes. C'est le cas, par exemple de Marie Dupuis, originaire de Montierneuf, admise à l'hôpital le 24 janvier 1783 et âgée de 48 ans ou de Marie Houdin, veuve d'un perruquier de Saint-Porchaire, et âgée de 41 ans. En outre, même dans le cas de femmes mariées, un mari dans l'incapacité de travailler conduit toute la famille au sein de l'institution, notamment lorsque celui-ci est un petit ouvrier cardeur ou un journalier payé à la tâche pour des travaux ingrats et pénibles. 
Pour autant, la pauvreté ne frappe pas indistinctement. Les pauvres ne se répartissent pas dans toutes les paroisses de la ville de façon harmonieuse. Au contraire, les données contenues dans les registres hospitaliers permettent d'établir une géographie de la pauvreté dans la ville, mais aussi les flux privilégiés de la pauvreté rurale échouant entre les murs de l'hôpital général.

\section{Origines géographiques}

Les tableaux qui suivent ont été construits grâce aux registres présentés supra ${ }^{14}$.

Tableau 4 - Origine paroissiale des pauvres de l'hôpital, 1659-1660 15

\begin{tabular}{|l|c|c|c|c|}
\hline Paroisses & Total VA & $\%$ & Dont femmes & $\%$ \\
\hline MONTIERNEUF & 9 & 23,7 & 3 & 33,3 \\
SAINTE-RADEGONDE & 8 & 21,1 & 7 & 87,5 \\
SAINT-GERMAIN & 1 & 2,6 & 0 & 0 \\
SAINTE-TRIAISE & 5 & 13,2 & 3 & 60 \\
SAINT-MICHEL & 6 & 15,8 & 1 & 33,3 \\
SAINT-PAUL & 1 & 2,6 & 1 & 33,3 \\
NOTRE-DAME-LA-PETITE & 1 & 2,6 & 1 & 33,3 \\
SAINT-PORCHAIRE & 1 & 2,6 & 1 & 33,3 \\
SAINT-CYBARD & 2 & 5,3 & 0 & 0 \\
LA CELLE & 1 & 2,6 & 1 & 33,3 \\
LA CUEILLE & 3 & 7,9 & 2 & 66,7 \\
\hline TOTAL & 38 & 100 & 21 & 55,3 \\
\hline
\end{tabular}

À la lecture du tableau 4, nous pouvons remarquer la prédominance des populations originaires de Montierneuf (23,7 \%), de Sainte-Radegonde (21,1 \%), de Saint-Michel (15,8 \%) et de Sainte-Triaise (13,2\%) au sein de l'hôpital. À elles seules, elles fournissent 73,8 \% des pauvres enfermés dans l'hôpital. Nous sommes donc en présence des paroisses les plus populaires de la ville de Poitiers. Ainsi, Montierneuf est-il un centre important du petit artisanat urbain. Les cardeurs et les journaliers en ont fait leur quartier. En outre, la paroisse de Sainte-Radegonde apparaît comme particulièrement habitée par des femmes pauvres : elles représentent $87,5 \%$ des admis de cette paroisse. Sans surprise, les paroisses plus " huppées " de Saint-Paul et de Notre-Dame-la-Petite ne sont que très peu représentées. Cette répartition change entre 1670 et 1679, mais de manière très relative.

14. Ici encore, les données ne s'inscrivent pas dans une stricte continuité chronologique. Les sources sont incomplètes.

15 . Origine non-connue pour $53,1 \%$ des admis. 
Emmanuel THÉVENET

Tableau 5 - Origine paroissiale des pauvres de l'hôpital, 1670-167916

\begin{tabular}{|l|c|c|c|c|}
\hline Paroisses & Total VA & $\%$ & Dont femmes & $\%$ \\
\hline MONTIERNEUF & 29 & 18,5 & 22 & 75,9 \\
SAINTE-RADEGONDE & 17 & 10,8 & 6 & 35,3 \\
SAINT-SATURNIN & 5 & 3,2 & 4 & 80 \\
SAINT-GERMAIN & 15 & 9,6 & 7 & 46,7 \\
SAINTE-TRIAISE & 11 & 7 & 8 & 72,7 \\
SAINT-MICHEL & 12 & 7,6 & 5 & 41,7 \\
SAINT-SULPICIEN & 7 & 4,5 & 4 & 57,1 \\
SAINT-PAUL & 2 & 1,3 & 1 & 50 \\
SAINT-DIDIER & 6 & 3,8 & 2 & 33,3 \\
NOTRE-DAME-LANCIENNE & 2 & 1,3 & 2 & 100 \\
NOTRE-DAME-LA-GRANDE & 1 & 0,6 & 1 & 100 \\
NOTRE-DAME-LA-PETITE & 5 & 3,2 & 3 & 60 \\
SAINT-PORCHAIRE & 11 & 7 & 5 & 45,5 \\
SAINT-CYBARD & 5 & 3,2 & 3 & 60 \\
LA CELLE & 6 & 3,8 & 3 & 50 \\
SAINT-PIERRE-L'HOUSTAULT & 2 & 1,3 & 0 & 0 \\
VOUNEUIL & 1 & 0,6 & 0 & 0 \\
AUMÔNERIE & 1 & 0,6 & 0 & 0 \\
SAINT-SAVIN & 5 & 3,2 & 4 & 80 \\
JACOBINS & 1 & 0,6 & 0 & 0 \\
SAINTE-OPPORTUNE & 8 & 5,1 & 5 & 62,5 \\
SAINT-ÉTIENNE & 2 & 1,3 & 1 & 50 \\
SAINT-SIMPLICIEN & 1 & 0,6 & 1 & 100 \\
SAINT-HILAIRE ÉGLISES & 1 & 0,6 & 0 & 0 \\
LA CHANDELIÈRE & 156 & 100 & 1 & 56,1 \\
\hline TOTAL & & & 88 & \\
\hline
\end{tabular}

Le tableau 5 met en évidence une certaine permanence de la pauvreté dans sa répartition paroissiale. En effet, Montierneuf, Sainte-Radegonde, SainteTriaise et Saint-Michel envoient à elles seules 44 \% des pauvres de l'hôpital. Néanmoins, cette proportion est en baisse par rapport à la période précédente. De fait, une progression de la pauvreté se fait jour : Saint-Germain est la paroisse d'origine de 9,6 \% des pauvres enfermés, et Sainte-Opportune de $5,1 \%$ de ceux-ci. Il y a donc un éparpillement de la pauvreté entre 1670 et 1679 , même si celui-ci reste relatif et temporaire. Néanmoins, il peut apparaître comme la marque des difficultés économiques de la fin du XVII e siècle, même si l'hôpital ne s'ouvre pas pour autant aux paroisses extérieures à la ville, à l'exception d'un pauvre, François Robé, admis le 20 novembre 1670 et originaire de Vouneuil, paroisse immédiatement voisine de Poitiers. La période qui suit constitue un retour à la situation de 1659-1660.

Tableau 6 - Origine paroissiale des pauvres de l'hôpital, 1700-1703 ${ }^{17}$

\begin{tabular}{|l|c|c|c|c|}
\hline Paroisses & Total VA & $\%$ & Dont femmes & $\%$ \\
\hline MONTIERNEUF & 32 & 19,5 & 32 & 100 \\
SAINTE-RADEGONDE & 26 & 15,9 & 15 & 57,7 \\
\hline
\end{tabular}

16. Origine non-connue pour $37,2 \%$ des admis.

17. Origine non-connue : $32,2 \%$ des admis. 


\begin{tabular}{|l|c|c|c|c|} 
SAINT-SATURNIN & 3 & 1,8 & 2 & 66,7 \\
SAINT-GERMAIN & 17 & 10,4 & 17 & 100 \\
SAINTE-TRIAISE & 6 & 3,7 & 6 & 100 \\
SAINT-MICHEL & 11 & 6,7 & 10 & 90,9 \\
SAINT-SIMPLICIEN & 2 & 1,2 & 1 & 50 \\
SAINT-PIERRE/PAUL & 4 & 2,4 & 2 & 50 \\
SAINT-DIDIER & 14 & 8,5 & 12 & 85,7 \\
NOTRE-DAME-LA-GRANDE & 4 & 2,4 & 4 & 100 \\
NOTRE-DAME-LA-PETITE & 4 & 2,4 & 4 & 100 \\
SAINT-PORCHAIRE & 11 & 6,7 & 11 & 100 \\
SAINT-CYBARD & 3 & 1,8 & 3 & 100 \\
LA CELLE & 4 & 2,4 & 4 & 100 \\
SAINT-PIERRE-L'HOUSTAULT & 1 & 0,6 & 1 & 100 \\
SAINT-ANDRÉ-QUINÇAY & 1 & 0,6 & 1 & 100 \\
SAINT-SAVIN & 3 & 1,8 & 3 & 100 \\
DOMINICALE & 4 & 2,4 & 2 & 50 \\
SAINTE-OPPORTUNE & 1 & 0,6 & 1 & 100 \\
SAINT-ÉTIENNE & 8 & 4,9 & 4 & 50 \\
SAINT-HILAIRE-CELLE & 3 & 1,8 & 2 & 66,7 \\
LA RÉSURRECTION & 2 & 1,2 & 2 & 84,8 \\
\hline TOTAL & 164 & 100 & 139 & \\
\hline
\end{tabular}

La similarité du tableau 6 avec celui de 1659-1660 est relative. En effet, le nombre de paroisses fournissant des contingents de pauvres à l'hôpital est plus important. Pour autant, leur répartition conforte la stratification sociale et la hiérarchisation géographique de Poitiers. Les paroisses populaires de Montierneuf, Sainte-Radegonde, Saint-Germain et Saint-Michel fournissent 52,5 \% des pauvres enfermés. Fait notable, Saint-Didier participe pour 8,5\% de cette population, et Saint-Etienne pour 4,9\% (contre $1,3 \%$ entre 1670 et 1673 et aucun admis pour la période 1659-1660). Mais Saint-Pierre-et-Paul, Notre-Dame-la-Grande et Notre-Dame-la-Petite sont les paroisses d'origine respectives de 2,4 \% des pauvres de l'hôpital. En outre, un seul pauvre n'est pas originaire de Poitiers : une femme originaire de Saint-André-de-Quinçay (paroisse rurale, relativement éloignée de la ville), admise le 23 novembre 1703 .

Entre 1710 et 1715, la situation se modifie franchement. Dans le premier quart du XVIII ${ }^{\mathrm{e}}$ siècle, l'hôpital général s'ouvre plus largement à la misère, accueillant notamment beaucoup plus de pauvres non-Poitevins.

Tableau 7 - Origine paroissiale des pauvres de l'hôpital, 1710-1715 ${ }^{18}$

\begin{tabular}{|l|c|c|c|c|}
\hline Paroisses & Total VA & $\%$ & femmes VA & $\%$ \\
\hline MONTIERNEUF & 36 & 15,9 & 30 & 83,3 \\
SAINTE-RADEGONDE & 20 & 8,8 & 14 & 70 \\
SAINT-SATURNIN & 1 & 0,4 & 1 & 100 \\
SAINT-GERMAIN & 9 & 4 & 8 & 88,9 \\
SAINTE-TRIAISE & 20 & 8,8 & 17 & 85 \\
SAINT-MICHEL & 7 & 3,1 & 6 & 85,7 \\
SAINT-SIMPLICIEN & 4 & 1,8 & 4 & 100
\end{tabular}

18. Origine non-connue : $26,8 \%$ des admis. 


\begin{tabular}{|l|c|c|c|c|} 
SAINT-PIERRE/PAUL & 5 & 2,2 & 5 & 100 \\
SAINT-DIDIER & 17 & 7,5 & 14 & 82,4 \\
NOTRE-DAME-ANCIENNE & 2 & 0,9 & 2 & 100 \\
NOTRE-DAME-LA-GRANDE & 3 & 1,3 & 3 & 100 \\
NOTRE-DAME-LA-PETITE & 14 & 6,2 & 11 & 78,6 \\
SAINT-PORCHAIRE & 15 & 6,6 & 12 & 80 \\
SAINT-CYBARD & 13 & 5,7 & 6 & 46,2 \\
LA CELLE & 11 & 4,8 & 9 & 81,8 \\
SAINT-PIERRE-L'HOUSTAULT & 1 & 0,4 & 1 & 100 \\
HÔTEL-DIEU & 5 & 2,2 & 3 & 60 \\
SAINT-SAVIN & 14 & 6,2 & 12 & 85,7 \\
DOMINICALE & 2 & 0,9 & 2 & 100 \\
SAINTE-OPPORTUNE & 1 & 0,4 & 1 & 100 \\
SAINT-ÉTIENNE & 6 & 2,6 & 5 & 83,3 \\
SAINT-HILAIRE-ÉGLISES & 2 & 0,9 & 2 & 100 \\
LA RÉSURRECTION & 4 & 1,8 & 3 & 75 \\
SAINT-SULPICIEN & 1 & 0,4 & 0 & 0 \\
AUMÔNERIE & 3 & 1,3 & 2 & 66,7 \\
SAINT-JEAN & 3 & 1,3 & 2 & 66,7 \\
LA CHANDELIÈRE & 3 & 1,3 & 3 & 100 \\
\hline SOUS-TOTAL & 222 & 97,8 & 178 & 80,2 \\
\hline LUSIGNAN & 1 & 0,4 & 1 & 100 \\
VOUNEUIL S/S BIARD & 1 & 0,4 & 0 & 0 \\
CHASSENEUIL & 1 & 0,4 & 1 & 100 \\
MONTMORILLON & 1 & 0,4 & 0 & 0 \\
CHATELLERAULT & 5 & 2,2 & 3 & $\mathbf{7 9 , 7}$ \\
\hline SOUS-TOTAL & $\mathbf{2 2 7}$ & $\mathbf{1 0 0}$ & $\mathbf{1 8 1}$ & \\
\hline TOTAL & & & 1 & 60 \\
\hline
\end{tabular}

Si 31,8 \% des pauvres de l'hôpital proviennent encore des paroisses (désormais bien connues) de Montierneuf, Sainte-Radegonde, Saint-Germain et Saint-Michel, leur part relative est en baisse importante par rapport à la période précédente (moins 20,7 points). On remarque l'augmentation de la proportion de pauvres issus de Saint-Didier, Sainte-Triaise, Saint-Porchaire, Saint-Cybard, dont la valeur s'établit à $28,6 \%$. Il y a donc un mouvement d'extension de la pauvreté, qui touche des paroisses jusqu'alors relativement épargnées. En outre, si l'écrasante majorité des pauvres est poitevine $(97,8 \%$ ), il y a tout de même une progression non négligeable de la proportion des pauvres originaires d'autres bourgs du Poitou (2,2\%). Surtout, ces individus viennent de plus loin : Vouneuil et Chasseneuil pour les plus proches, Châtellerault, Montmorillon et Lusignan pour les plus éloignés, présence d'autant plus problématique qu'à l'exception de Chasseneuil et de Vouneuil, toutes ces villes disposent d'un hôpital. Leur présence peut dès lors s'expliquer par deux hypothèses : soit les hôpitaux locaux sont surchargés et les pauvres surnuméraires sont envoyés à Poitiers (mais dans ce cas, pourquoi chaque cité n'en envoie-t-elle qu'un seul?), soit il s'agit de pauvres errants, originaires de ces villes, mais que la misère a poussés sur les routes jusqu'à la capitale provinciale, où les chasse-coquins les ont capturés. Cette dernière interprétation semble la plus satisfaisante.

La période suivante (1715-1722) confirme le mouvement de la pauvreté. 
La pauvreté en Poitou sous l'Ancien Régime

Tableau 8 - Origine paroissiale des pauvres de l'hôpital, 1715-1722 ${ }^{19}$

\begin{tabular}{|l|c|c|c|c|}
\hline Paroisses & Total VA & $\%$ & Dont femmes & $\%$ \\
\hline MONTIERNEUF & 71 & 21,3 & 53 & 74,6 \\
SAINTE-RADEGONDE & 19 & 5,7 & 15 & 78,9 \\
SAINT-SATURNIN & 4 & 1,2 & 3 & 75 \\
SAINT-GERMAIN & 29 & 8,7 & 19 & 65,5 \\
SAINTE-TRIAISE & 19 & 5,7 & 18 & 94,7 \\
SAINT-MICHEL & 16 & 4,8 & 13 & 81,3 \\
SAINT-SIMPLICIEN & 4 & 1,2 & 4 & 100 \\
SAINT-PIERRE/PAUL & 8 & 2,4 & 6 & 75 \\
SAINT-DIDIER & 43 & 12,9 & 35 & 81,4 \\
NOTRE-DAME-LA-GRANDE & 3 & 0,9 & 1 & 33,3 \\
NOTRE-DAME-LA-PETITE & 10 & 3 & 6 & 60 \\
SAINT-PORCHAIRE & 30 & 9 & 22 & 73,3 \\
SAINT-CYBARD & 18 & 5,4 & 14 & 77,8 \\
LA CELLE & 7 & 2,1 & 2 & 28,6 \\
SAINT-PIERRE-L'HOUSTAULT & 15 & 4,5 & 9 & 60 \\
HÔTEL-DIEU & 1 & 0,3 & 0 & 0 \\
SAINT SAVIN & 10 & 3 & 8 & 80 \\
DOMINICALE & 2 & 0,6 & 0 & 0 \\
SAINTE-OPPORTUNE & 4 & 1,2 & 3 & 75 \\
SAINT-ÉTIENNE & 1 & 0,3 & 1 & 100 \\
SAINT-HILAIRE ÉGLISES & 6 & 1,8 & 5 & 83,3 \\
LA RÉSURRECTION & 3 & 0,9 & 3 & 100 \\
SAINT-SULPICIEN & 5 & 1,5 & 5 & 100 \\
SAINT-JEAN & 2 & 0,6 & 1 & 50 \\
\hline SOUS-TOTAL & 1 & 98,8 & 246 & 74,5 \\
\hline JRAYS & 1 & 0,3 & 1 & 100 \\
BLOIS & $\mathbf{3 3 4}$ & 0,3 & 1 & 100 \\
PARIS & 0,3 & 1 & $\mathbf{7}$ \\
COULOMBIERS & 0,3 & $\mathbf{7 4 , 9}$ \\
\hline SOUS-TOTAL & 1,2 & $\mathbf{2 5 0}$ & \\
\hline TOTAL & 100 & & 100 \\
\hline
\end{tabular}

Il apparaît que, au cours du XvIII siècle, la pauvreté s'insinue profondément dans les paroisses de la ville de Poitiers nouvellement conquises. Les quatre paroisses populaires étudiées auparavant ne fournissent plus que 40,5 \% des pauvres de l'hôpital. Cependant si Saint-Didier voit la part de ses ressortissants passer à 12,9\%, Saint-Porchaire voit la sienne s'établir à $9 \%$. En outre, si la proportion de pauvres non-poitevins augmente d'un point $(1,2 \%)$, leurs villes d'origine sont plus éloignées que précédemment. Paris, Blois, Jrays (?) et Coulombiers ont chacune un représentant à l'hôpital général, probablement des errants, à l'exception de deux femmes, entrées à l'hôpital de Poitiers le 10 février 1719, l'une, parisienne, déplacée de la Conciergerie à l'hôpital par décision de la maréchaussée, l'autre, âgée de 6 ans, venue de Blois, mais née à Strasbourg d'un père soldat. La fin du XVIII ${ }^{\mathrm{e}}$ siècle confirme cette tendance d'ouverture.

19. Origine non-connue : $26,6 \%$ des admis. 
Tableau 9 - Origine paroissiale des pauvres de l'hôpital, Poitevins seuls, 1777-1789 20

\begin{tabular}{|l|c|c|c|c|}
\hline Paroisses & Total VA & $\%$ & Dont femmes & $\%$ \\
\hline MONTIERNEUF & 109 & 13 & 58 & 53,2 \\
SAINTE-RADEGONDE & 76 & 9,1 & 41 & 53,9 \\
LA RÉSURRECTION & 24 & 2,9 & 10 & 41,7 \\
SAINT-SATURNIN & 37 & 4,4 & 19 & 51,4 \\
SAINT-GERMAIN & 93 & 11,1 & 57 & 61,3 \\
SAINTE-TRIAISE & 45 & 5,4 & 25 & 55,6 \\
SAINT- MICHEL & 50 & 6 & 28 & 56 \\
SAINT-PAUL & 5 & 0,6 & 4 & 80 \\
SAINT-DIDIER & 72 & 8,6 & 48 & 66,7 \\
HÔTEL-DIEU & 3 & 0,4 & 2 & 66,7 \\
NOTRE-DAME-LA-GRANDE & 3 & 0,4 & 3 & 100 \\
NOTRE-DAME-LA-PETITE & 16 & 1,9 & 11 & 68,8 \\
SAINT-PORCHAIRE & 55 & 6,6 & 26 & 42 \\
SAINT-CYBARD & 17 & 2 & 8 & 47,1 \\
LA CELLE & 17 & 2 & 11 & 64,7 \\
SAINT-AUSTRÉGÉSILE & 3 & 0,4 & 1 & 33,3 \\
SAINT-SAVIN & 8 & 1 & 6 & 75 \\
NOTRE-DAME-DES-CHAMPS & 1 & 0,1 & 0 & 0 \\
SAINTE-OPPORTUNE & 8 & 1 & 5 & 62,5 \\
SAINT-ÉTIENNE & 6 & 0,7 & 4 & 66,7 \\
SAINT-SIMPLICIEN & 20 & 2,4 & 11 & 55 \\
SAINT-HILAIRE-ÉGLISES & 4 & 0,5 & 4 & 100 \\
LA CHANDELIĖRE & 5 & 0,6 & 3 & 60 \\
\hline SOUS-TOTAL & 709 & 84,5 & 385 & 54,3 \\
\hline
\end{tabular}

En effet, la fin du xvIII ${ }^{\mathrm{e}}$ siècle est marquée par deux phénomènes. À Poitiers, les quatre paroisses pauvres sont à l'origine de $39,2 \%$ des individus hospitalisés. En ce qui concerne les paroisses plus nouvellement pauvres que sont Saint-Didier et Saint-Porchaire, le cumul de leurs représentants forme 15,2 \% des pauvres de l'hôpital, soit un recul de 6,7 points. La pauvreté semble donc se stabiliser à la veille de la Révolution, dans des paroisses accueillant des individus issus des classes populaires et de la "lower middle class".

Le second phénomène tient à la forte croissance du taux de pauvres issus de paroisses étrangères à Poitiers. Entre 1777 et 1789, les paroisses poitevines ne représentent " que " $84,5 \%$ des pauvres. Le tableau 10 met en évidence la grande diversité géographique des pauvres admis à l'hôpital général de Poitiers.

Tableau 10 - Origine paroissiale des pauvres de l'hôpital, non-Poitevins seuls, 1777-1789

\begin{tabular}{|l|c|c|c|c|}
\hline Paroisses & Total VA & $\%$ & Dont femmes & $\%$ \\
\hline MARNAY & 2 & 0,2 & 2 & 100 \\
BIARD & 2 & 0,2 & 0 & 0 \\
\hline
\end{tabular}

20. Origine non-connue : $4 \%$ des admis. 
La pauvreté en Poitou sous l'Ancien Régime

\begin{tabular}{|c|c|c|c|c|}
\hline MARÇAY & 4 & 0,5 & 2 & 50 \\
\hline SAINT-BENOÎT & 5 & 0,6 & 2 & 40 \\
\hline NOUAILLE & 13 & 1,6 & 7 & 53,8 \\
\hline JOURNET & 1 & 0,1 & 1 & 100 \\
\hline JAUNAY & 8 & 1 & 1 & 12,5 \\
\hline CHÂTEAU-LARCHER & 5 & 0,6 & 2 & 40 \\
\hline SAVIGNY & 1 & 0,2 & 0 & 0 \\
\hline CHASSENEUIL & 7 & 0,9 & 5 & 71,4 \\
\hline THOUARS & 5 & 0,6 & 0 & 0 \\
\hline CISSE & 8 & 1 & 3 & 37,5 \\
\hline SAINT-SAVIN & 8 & 1 & 6 & 75 \\
\hline VAUX & 1 & 0,1 & 0 & 0 \\
\hline MIGNE & 7 & 0,9 & 3 & 42,9 \\
\hline MIGNALOUX & 2 & 0,2 & 0 & 0 \\
\hline SAINT-CYR & 1 & 0,1 & 1 & 100 \\
\hline CHARROUX & 1 & 0,1 & 0 & 0 \\
\hline VOUILLÉ & 1 & 0,1 & 0 & 0 \\
\hline BOURESSE & 1 & 0,1 & 1 & 100 \\
\hline BÉRUGES & 9 & 1,1 & 3 & 33,3 \\
\hline SMARVES & 3 & 0,4 & 0 & 0 \\
\hline PARTHENAY & 10 & 1,2 & 4 & 40 \\
\hline ARGENTON & 2 & 0,2 & 0 & 0 \\
\hline CURZAY & 1 & 0,1 & 1 & 100 \\
\hline DISSAY & 3 & 0,4 & 0 & 0 \\
\hline BICÊTRE & 1 & 0,1 & 1 & 100 \\
\hline LATILLE & 1 & 0,1 & 1 & 100 \\
\hline MONTMORILLON & 1 & 0,1 & 0 & 0 \\
\hline SEVRES & 1 & 0,1 & 1 & 100 \\
\hline ANXAUMONT & 1 & 0,1 & 0 & 0 \\
\hline SANXAY & 1 & 0,1 & 0 & 0 \\
\hline CHARRAIS & 1 & 0,1 & 1 & 100 \\
\hline CELLE-L'ÉVESCAULT & 1 & 0,1 & 1 & 100 \\
\hline QUINÇAY & 2 & 0,2 & 1 & 50 \\
\hline ANDILLE & 2 & 0,2 & 0 & 0 \\
\hline AIRVAULT & 1 & 0,1 & 0 & 0 \\
\hline LE BLANC & 1 & 0,1 & 1 & 100 \\
\hline CHIRÉ-LES BOIS & 1 & 0,1 & 0 & 0 \\
\hline COUHÉ & 2 & 0,2 & 1 & 50 \\
\hline SAINT-LAON & 1 & 0,1 & 0 & 0 \\
\hline SANXAY & 1 & 0,1 & 0 & 0 \\
\hline SOUS-TOTAL & 130 & 15,5 & 52 & 40 \\
\hline TOTAL & 839 & 100 & 437 & 54,2 \\
\hline
\end{tabular}

Ainsi, l'hôpital s'ouvre-t-il à la pauvreté de nombreuses paroisses rurales. En tout, ces pauvres non-poitevins représentent 15,5 \% de la totalité des pauvres enfermés. Pour autant, leurs origines sont très éclatées. La paroisse la plus représentée est Nouaillé, qui fournit 1,6 \% des pauvres, suivie de Parthenay, qui est à l'origine de 1,2 \% d'entre eux. Il est remarquable que les pauvres de Nouaillé semblent profiter d'un accord de partenariat, issu du rattachement de leur aumône à l'hôpital général. En ce qui concerne les pauvres de Parthenay, leur présence à Poitiers est plus problématique, le bourg de Gâtine disposant d'un hôpital.

Au final, les caractéristiques géographiques de la pauvreté admise à l'hôpital général de Poitiers sont doubles. D'une part, on constate la forte pré- 
éminence de la pauvreté urbaine et poitevine au sein de l'établissement. À l'intérieur de la cité, les paroisses les plus pauvres sont également les plus populaires, et conservent, sur cent trente années, leur qualité de fournisseurs principaux. Pour autant, la pauvreté n'est pas figée dans la ville. Au fil du temps, de nouvelles paroisses sont touchées, de façon profonde. D'autre part, si dans un premier temps l'hôpital général de Poitiers semble s'inscrire uniquement dans sa mission initiale, c'est-à-dire accueillir la pauvreté et la mendicité poitevines, à partir de 1715 et surtout de 1748 , la structure s'ouvre. Elle abrite à la fois des pauvres issus de la province de Poitou et des individus venant de villes plus lointaines (Paris, Blois), voire des étrangers (Irlandais, notamment, tels que Jacques MacHabee, ou Jean Bourré, originaire de la ville de Sat, tous les deux morts à l'hôpital dans le premier tiers du xvIII siècle).

Pour compléter ce tableau de la pauvreté, une étude des caractères sociaux de celle-ci s'impose.

\section{Origines sociales}

Pour déterminer l'identité sociale des pauvres enfermés, nous étudierons leurs professions ou qualités, ainsi que leur état matrimonial. Le graphique qui suit a été construit grâce aux données contenues dans les registres de sépultures de l'hôpital ${ }^{21}$. Nous n'avons pas pu utiliser les registres des délibérations du bureau, en raison de la trop faible proportion d'individus dont la profession est connue. Dès lors, cette stratification sociale n'est valable que pour le premier tiers du XvIII siècle.

La lecture du graphique 6 révèle la forte présence des journaliers, cardeurs et tisserands au sein de la population pauvre enfermée. Ces trois groupes réunis représentent près de $42 \%$ de la totalité des pauvres renfermés. La forte présence des journaliers s'explique par la précarité structurelle de cette profession, constituée de petits ouvriers ou artisans se louant à la journée ou pour de très courtes durées.

Les deux autres professions fortement minoritaires donnent, quant à elles, une indication de l'état économique de la ville de Poitiers au début du XvIII ${ }^{\mathrm{e}}$ siècle. Il apparaît que cardeurs et tisserands sont victimes d'une crise qui touche la proto-industrie textile poitevine. Cette crise est en général attribuée à la médiocre qualité des tissus produits, comparés à ceux de Lyon ou de la Bretagne, et à une inadéquation des productions avec les modes nouvelles, telles que les indiennes de coton. Dès lors, l'hôpital joue le rôle de régulateur économique évoqué auparavant, en participant à la résorption du chômage, tout en assurant une production " publique ", par le biais de la manufacture de tissus. Par ailleurs, cette crise semble toucher la branche " textile " en son entier, puisque les sergetiers et les bonnetiers, auxquels nous pouvons ajouter les tailleurs d'habits, représentent 6,5\% des pauvres morts à l'hôpital.

21. Arch. mun. de Poitiers (médiathèque François-Mitterrand), registres de sépultures 270 et 271. 
Graphique 6 - Origines socioprofessionnelles des morts à l'hôpital, 1704-1730

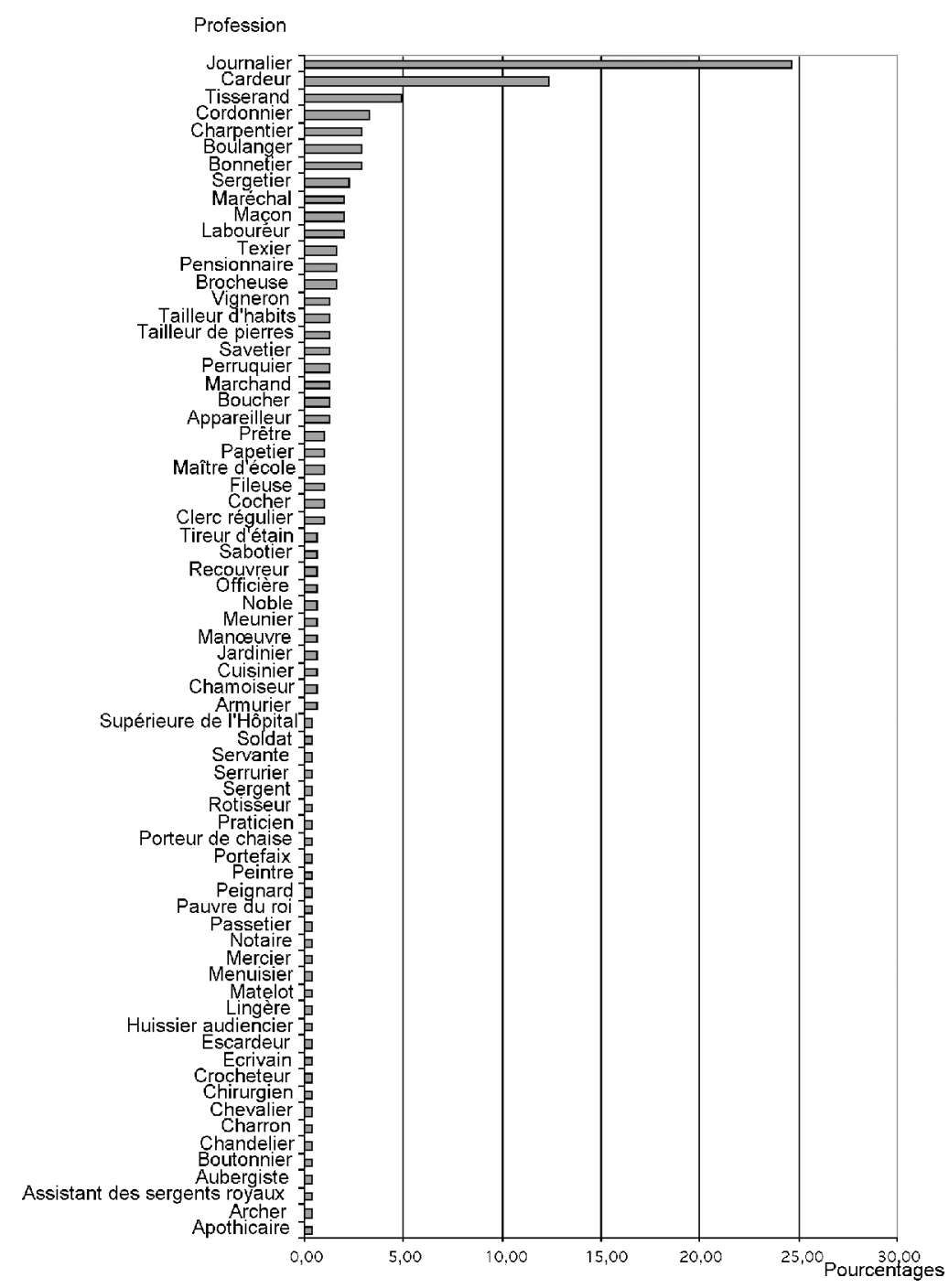

De manière globale, ce graphique permet de prendre conscience des très nombreuses professions de l'Ancien Régime, mais aussi de la grande diversité des métiers touchés, ponctuellement ou non, par une pauvreté telle que la seule solution réside dans l'enfermement. Pour autant, très peu de professions "supérieures " sont représentées au sein de l'hôpital : nous remarquons un fils de notaire royal, Antoine Leblonc, originaire de Chartres, et un chevalier (de la Coindrie), Alexis Richeteau Equier, âgé de 
45 ans et originaire de Thouars. Par ailleurs, certaines professions représentées sont surprenantes, comme celle de matelot : ainsi, Pierre Gautier, âgé de 28 ans et né à Bordeaux, échoue-t-il à l'hôpital général de Poitiers, relativement loin de la côte.

En ce qui concerne les caractéristiques matrimoniales des individus enfermés, nous pouvons surtout travailler sur la notion de veuvage, notamment féminin. En effet, les informations contenues dans les registres des délibérations mettent en évidence l'importante proportion de veuves mais signalent de façon beaucoup plus ponctuelle le veuvage masculin.

Le tableau suivant, construit d'après les données des registres de délibérations et des registres des sépultures, montre la part relative des veuves dans la population féminine de l'hôpital.

Tableau 12 - Proportion de veuves à l'hôpital général, 1670-178922

\begin{tabular}{|l|c|c|c|c|c|c|c|c|}
\hline Années & $1670-1673$ & $1673-1674$ & $1674-1675$ & $1675-1679$ & $1709-1730$ & $1748-1755$ & $1777-1785$ & $1786-1789$ \\
\hline Femmes VA & 89 & 29 & 30 & 33 & 795 & 377 & 520 & 182 \\
\% de veuves & 4,5 & 0 & 0 & 12,1 & 18,1 & 9,3 & 18,3 & 19,2 \\
\hline
\end{tabular}

Le tableau 12 met en évidence la rupture forte qu'institue la fin du $\mathrm{XVII}^{\mathrm{e}}$ siècle dans le rapport des femmes au veuvage. Les veuves sont en effet absentes de l'hôpital pendant trois ans, après avoir représenté 4,5 \% des femmes enfermées. Mais, à partir de 1675, cette proportion oscille entre 9,3 et $19,2 \%$. Il y a donc une modification profonde du destin des veuves poitevines dans le dernier quart du XVII ${ }^{\mathrm{e}}$ siècle. Celle-ci peut apparaître comme le résultat d'un durcissement de la législation royale les concernant.

En effet, les veuves, soumises à des lois civiles spécifiques, s'appliquant notamment à l'administration de leurs biens, voire à la direction d'ateliers artisanaux, jouissent d'une relative autonomie dans une société fortement patriarcale et phallocratique. Cette autonomie est particulièrement inquiétante pour la monarchie, notamment dans la mesure où le capital des veuves peut passer dans des mains étrangères, et ce, sans l'intervention de la famille. Dès lors, les mesures visant à restreindre le champ d'action des femmes sans mari peuvent probablement les conduire à demeurer seules, et par conséquent à finir leurs jours à l'hôpital général.

À l'issue de cette étude, des conclusions peuvent être dégagées. La pauvreté a une identité fluctuante, mais celle-ci s'établit autour d'un portraittype qu'il faut toujours retoucher pour être exact. La pauvreté est donc plutôt âgée, féminine, artisanale ou manouvrière et assez fréquemment touchée par le veuvage. Pour autant, cela n'exclut pas la présence au sein de l'hôpital d'hommes plus jeunes et plus vigoureux. Par ailleurs, quelle que soit l'identité sociale de ces pauvres, une constante se révèle : l'essentiel des pauvres a une origine urbaine. En outre, les variations du

22. Les différentes périodes considérées correspondent aux sources disponibles. 
nombre de pauvres enfermés à l'hôpital semblent tenir autant à des causes structurelles qu'à des évolutions de l'environnement économique ou frumentaire. La maxime scolastique "post hoc, ergo propter hoc ${ }^{23}$ " se révèle exacte dans son inexactitude. Par ailleurs, la population de la " pauvre Babylone $^{24}$ " que forme l'hôpital ne se limite pas aux pauvres : ceux-ci sont encadrés par un " personnel hospitalier " nombreux et divers, qui regroupe des clercs et des laïcs, des hommes et des femmes, des nobles et des roturiers, et qui mériterait de faire l'objet d'une étude spécifique.

Enfin, l'analyse qui précède tend à occulter par son objet même un pan important de la pauvreté sous l'Ancien Régime. En effet, l'étude de la population pauvre pour elle-même avec pour base de travail les données fournies par les documents que l'hôpital a produits, donne l'impression, fausse, d'une institution performante, normative, voire totalitaire dans sa démarche. En réalité, l'hôpital général, tout au long de son existence se trouve aux prises avec de graves problèmes de gestion, d'origine structurelle et conjoncturelle. Initialement prévu pour accueillir trois cents personnes, il doit parfois abriter plus de quatre cents pauvres. En outre, la gestion mise en place par les administrateurs, et empreinte de prodigalité, nuit à l'équilibre de l'ensemble. Comme le relève Dansay, le président des trésoriers de France, le 2 mai 1780 : «Le revenu est insuffisant pour nourrir le nombre de pauvres dont l'hôpital est surchargé [...] On a écrit aux hôpitaux voisins pour savoir quels moyens ils avaient employés pour soutenir leurs maisons [...]; les hôpitaux ont les uns $150 \mathrm{~L}$ et les autres $130 \mathrm{~L}$, les moins à leur aise $120 \mathrm{~L}$ par teste pour chacun de leurs pauvres, tandis que chaque individu de cette maison n'a pas $45 \mathrm{~L}$ à dépenser par an ${ }^{25}$. " Ainsi, l'hôpital général de Poitiers ne dispose pour chaque pauvre que du tiers de ce que consacrent aux leurs les établissements les plus riches. Dans de telles conditions, il n'est pas étonnant qu'il soit fréquemment au bord du gouffre financier : en septembre 1697, la sœur Gendreau vient au bureau pour expliquer que les pauvres sont presque nus, et qu'à l'approche de l'hiver, il est nécessaire de les vêtir. En 1709, année du " grand hyver ", l'intendant écrit au contrôleur général pour l'aviser de la situation de la ville de Poitiers : « [...] Le souci avec lequel on s'occupe des deux mille trois ou quatre cents pauvres placés dans les hôpitaux à la charge de la ville fait que celle-ci se trouve dans la plus parfaite tranquillité ${ }^{26}$. " Mais rappelons que Poitiers compte à l'époque quelque vingt mille habitants, que les pauvres représentent par conséquent $11,5 \%$ de la population, et que la tranquillité publique n'est obtenue qu'au prix d'impositions exceptionnelles. En 1737, les administrateurs en appellent à l'intendant, en raison du fort nombre de pauvres et de la cherté des grains, et du refus de l'évêque d'honorer les rentes ecclésiastiques. Lenain doit donc intervenir dans le

23. "À la suite de cela, donc à cause de cela".

24. L'expression est du père Louis-Marie Grignion de Montfort.

25. Cité par RamBaud, Pierre, L'Assistance publique..., op. cit., p. 540-541.

26. Paultre, Christian, La Répression de la mendicité et du vagabondage sous l'Ancien régime, Paris, 1906. 
procès qui oppose l'hôpital au clergé. À nouveau, en 1741, la situation se dégrade et oblige le bureau à contracter un emprunt de six mille livres afin de nourrir les pauvres enfermés ${ }^{27}$. Cette situation chaotique se prolonge pendant la Révolution. Il semble que les hommes de l'Ancien Régime, comme ceux qui leur succèdent à l'époque contemporaine, aient durablement échoué dans leur tentative d'éradication de la pauvreté. Malgré la diversité des techniques employées (aumôneries médiévales, hôpitaux généraux de l'âge classique, prise en charge à domicile pendant la Révolution, maisons d'enfermement, workhouses, hôpitaux psychiatriques et prisons de l'époque contemporaine), la société ne parvient pas à détruire la pauvreté, probablement en raison d'une lecture par trop partiale et partielle de celle-ci, mettant en avant des critères économiques aux dépens d'une approche morale et philosophique.

\section{RÉSUMÉ}

Fondé en 1657, l'Hôpital général de Poitiers accueille une population majoritairement féminine, issue du petit salariat agricole ou de l'artisanat textile, vieillissante au fur et à mesure que l'on avance dans le XVIII ${ }^{\mathrm{e}}$ siècle. Initialement destiné à rééduquer par le travail les pauvres et les mendiants de la ville, l'établissement recrute de plus en plus dans des quartiers centraux et aisés du plateau, signe d'une extension de la misère, et s'ouvre également vers la fin du siècle à une population étrangère, originaire de paroisses rurales, parfois extérieures à la province.

\section{ABSTRACT}

Founded in 1657, the Hôpital Général of Poitiers welcomes a largely feminine population, which comes from small agricultural wage-earning people or textile craftsmanship, getting older as the $18^{\text {th }}$ Century proceeds. Initially built to re-educate poor people and beggars from the town by working, the institution recruits more and more in middle and well-to-do quarters of the plateau, sign of the extension of misery, and at the end of the century, opens itself to a foreign population originating from rural parishes, sometimes outside of the province.

27. RAmBAUD, Pierre, L'Assistance publique ..., op cit., p. 538-541. 